\title{
Investigation of Maximum Blade Loading Capability of Lift-Offset Rotors
}

\author{
Hyeonsoo Yeo \\ hyeonsoo.yeo.civ@mail.mil \\ US Army Aviation Development Directorate - AFDD \\ Aviation \& Missile Research, Development \& Engineering Center \\ Research, Development, and Engineering Command \\ Ames Research Center, Moffett Field, California \\ Wayne Johnson \\ wayne.johnson@nasa.gov \\ Aeromechanics Branch \\ National Aeronautics and Space Administration \\ Ames Research Center, Moffett Field, California
}

\begin{abstract}
Maximum blade loading capability of a coaxial, lift-offset rotor is investigated using a rotorcraft configuration designed in the context of short-haul, medium-size civil and military missions. The aircraft was sized for a 6600-lb payload and a range of $300 \mathrm{~nm}$. The rotor planform and twist were optimized for hover and cruise performance. For the present rotor performance calculations, the collective pitch angle is progressively increased up to and through stall with the shaft angle set to zero. The effects of lift offset on rotor lift, power, controls, and blade airloads and structural loads are examined. The maximum lift capability of the coaxial rotor increases as lift offset increases and extends well beyond the McHugh lift boundary as the lift potential of the advancing blades are fully realized. A parametric study is conducted to examine the differences between the present coaxial rotor and the McHugh rotor in terms of maximum lift capabilities and to identify important design parameters that define the maximum lift capability of the rotor. The effects of lift offset on rotor blade airloads and structural loads are also investigated. Flap bending moment increases substantially as lift offset increases to carry the hub roll moment even at low collective values. The magnitude of flap bending moment is dictated by the lift-offset value (hub roll moment) but is less sensitive to collective and speed.
\end{abstract}

\section{NOTATION}

speed of sound

rotor disk area, $\pi R^{2}$

blade chord

rotor lift coefficient, $L / \rho(\Omega R)^{2} A$

rotor power coefficient, $P / \rho(\Omega R)^{3} A$

rotor induced power coefficient, $P_{i} / \rho(\Omega R)^{3} A$

rotor profile power coefficient, $P_{o} / \rho(\Omega R)^{3} A$

rotor thrust coefficient, $T / \rho(\Omega R)^{2} A$

rotor diameter

section lift per unit length

rotor lift

section Mach number

section lift coefficient, $l / \frac{1}{2} \rho a^{2} c$

number of blades

rotor power

rotor induced power
$P_{o} \quad$ rotor profile power

Presented at the AHS 69th Annual Forum, Phoenix, Arizona, May 21-23, 2013. This is a work of the U.S. Government and is not subject to copyright protection in the U.S. $q \quad$ dynamic pressure

$R \quad$ rotor radius

$T \quad$ rotor thrust

$V_{T I P} \quad$ rotor blade tip speed

$V_{\infty} \quad$ free-stream velocity

$X \quad$ rotor propulsive force

$\alpha_{s} \quad$ geometric shaft angle (positive for rearward tilt)

$\theta_{o} \quad$ collective, deg

$\theta_{1 c} \quad$ lateral cyclic, deg

$\theta_{1 s} \quad$ longitudinal cyclic, deg

$\mu \quad$ advance ratio, $V_{\infty} / \Omega R$

$\rho \quad$ free-stream density

$\sigma \quad$ solidity

$\Omega \quad$ rotor angular rotation rate

\section{INTRODUCTION}

Emerging military and commercial needs are leading to requirements for significant increases in speed and range capabilities over what conventional helicopters can achieve. The high-speed performance of conventional helicopters is significantly limited by adverse effects of compressibility on the ad- 
vancing blades and stall on the retreating blades of the rotor. Lift capability of the retreating blades decreases with forward speed due to low dynamic pressure, reverse flow, and stall. As a result, the advancing blades are not allowed to generate much lift if roll moment trim is to be maintained. Thus, the advancing blades operate at non-optimum angles of attack, resulting in poor rotor performance. By operating a rotor in edgewise flight with lift offset - more lift on the advancing side than on the retreating side of the rotor disk - attaining good performance at high forward speeds is possible. The lift potential of the advancing blades can be fully realized and retreating blade stall can be avoided or minimized. The rotor rotation speed must be slowed as the flight speed increases, in part to minimize the compressible drag rise on the advancing blades.

The lift-offset concept was demonstrated by the XH-59A Advancing Blade Concept (ABC) demonstrator aircraft during the 1970s (Ref. 1). The XH-59A's ABC system consisted of two three-bladed, coaxial, counter-rotating rigid rotors. With the auxiliary turbojets, the XH-59A achieved a maximum level speed of 240 knots at 3000-ft altitude and a maximum speed of 263 knots in a 7-deg dive at $13000-\mathrm{ft}$ altitude (Ref. 2). The high lift capability of the ABC rotor system was also shown in the XH-59A flight tests for both steady level flight and transient maneuvers, including nondimensional blade loading up to $C_{T} / \sigma=0.28$ in maneuvering flight (Ref. 3). Despite the high-speed, high-lift capabilities of the XH-59A, the flight test revealed technical challenges such as poor aerodynamic performance, high rotor weight, hub drag, vibration, etc. With recent interest in high-speed rotorcraft, Sikorsky Aircraft has incorporated several new technologies (high lift-to-drag ratio rigid blades, low drag hub fairings, Active Vibration Control (AVC), integrated auxiliary propulsion system, and fly-by-wire flight controls) into the X2 Technology ${ }^{\mathrm{TM}}$ Demonstrator aircraft and has successfully demonstrated them in a flight environment (Refs. 4-7).

Recently NASA and the U.S. Army at Ames Research Center conducted research on lift-offset rotorcraft to explore the performance potential of modern-technology lift-offset rotors and to better understand the impact of key technologies on rotor performance, weight, and airframe aerodynamics of rotorcraft with lift-offset rotors (Refs. 8,9). These studies also established aerodynamic modeling requirements for performance calculations and designed lift-offset rotorcraft for short-haul, medium-size civil and military missions.

The present study explores the high blade loading capabilities demonstrated by the XH-59A flight test using a moderntechnology lift-offset rotor. The objective of this paper is to better understand and quantify high blade loading $\left(C_{T} / \sigma\right)$ capability of a coaxial, lift-offset rotor and ultimately establish blade loading design guidance for maneuver capability. A parametric study is conducted to examine the differences between the present coaxial rotor and the $\mathrm{McHugh}$ rotor in terms of maximum lift capabilities and to identify important design parameters that define the maximum lift capability of the rotor. The effects of lift offset on rotor blade airloads and struc- tural loads are also investigated.

\section{COAXIAL, LIFT-OFFSET ROTORCRAFT}

The configuration of the coaxial, lift-offset rotorcraft for the present study is shown in Fig. 1. The present coaxial rotorcraft is one of the four configurations that were designed in the context of short-haul, medium-size civil and military missions (Ref. 9). The aircraft was sized for a 6600 -lb payload and a range of $300 \mathrm{~nm}$. The basic characteristics of the aircraft are shown in Table 1. The aircraft has two main rotors in a coaxial configuration, a pusher propeller for cruise propulsion, and horizontal and vertical tails for cruise trim. The aircraft uses stiff coaxial main rotors capable of carrying significant roll moment, hence generating lift on the rotor advancing side in forward flight. The aircraft requires auxiliary propulsion at high speeds, but has no wing. The upper rotor rotates counter-clockwise, whereas the lower rotor rotates clockwise when viewed from the top. The vertical separation distance between the two rotors is $7 \%$ rotor diameter.

The hingeless blade inertial and structural properties were scaled from the compound helicopter blade design of Ref. 10. State-of-the-art rotor airfoils were used for the main rotor blades. The rotor planform and twist were optimized for hover and cruise performance. A two-parameter twist distribution was considered: linear twist inboard and outboard of $0.5 \mathrm{R}$. A three-parameter taper distribution was considered: linear taper from 0 to $0.35 R$, from $0.35 R$ to $0.75 R$, and from $0.75 R$ to the tip. Here taper ratio is defined as the ratio of tip chord to root chord. The inboard taper ratio was fixed at 1.66, based on structural considerations. The optimum rotor geometry determined from a trade between hover and cruise efficiency is: linear twist rate $=-6^{\circ}$ inboard and $-12^{\circ}$ outboard; linear taper ratio $=1.66$ inboard, 1.3 midspan, and 0.1 outboard. The optimum rotor geometry from a trade between hover and cruise efficiency is shown in Fig. 2.

\section{MODELING AND ANALYSIS}

The rotor performance calculations were carried out using the comprehensive rotorcraft analysis CAMRAD II (Ref. 11). CAMRAD II is an aeromechanics analysis of rotorcraft that incorporates a combination of advanced technologies including multibody dynamics, nonlinear finite elements, and rotorcraft aerodynamics. CAMRAD II has been used extensively for correlation of performance and loads measurements of several rotors operating in various flight conditions (Refs. 12-14). The coaxial, lift-offset rotor was modeled in CAMRAD II, but not the airframe or propeller. The CAMRAD II aerodynamic model for the rotor blade is based on lifting line theory, using steady two-dimensional airfoil characteristics and a vortex wake model. Rotor performance was calculated using nonuniform inflow with rigid wake geometry and unsteady aerodynamics, but a dynamic stall model was not used. Six beam elements were used in modeling a main rotor blade and 18 aerodynamic segments were used for aerodynamic calculation, with a root cutout of $0.12 \mathrm{R}$. Airfoil characteristics were obtained from C81 tables. 
For this calculation, the collective pitch angle was progressively increased up to and through stall with the shaft angle set to zero. The trim solution specifies zero hub pitching moment for each rotor and equal but opposite values for hub rolling moment for each rotor. The trim variables are longitudinal and lateral cyclics of both rotors. In this calculation, both rotors operate at the same collective. However, torque balance was not required. This approach is an approximate way of looking at maneuver capability.

The present analysis approach was used to predict the aerodynamic rotor lift boundary obtained in a wind tunnel test of a model rotor (Ref. 15). The correlation results will be presented in the following section.

\section{RESULTS AND DISCUSSION}

In this section, the performance and loads of coaxial, liftoffset rotors are presented to better understand and quantify the high blade-loading capability of this configuration. The effects of lift-offset on rotor blade airloads and structural loads are also shown.

\section{Performance}

Figure 3 shows the performance of coaxial, lift-offset rotors. The total rotor lift is plotted as a function of the sum of induced and profile power of both rotors (for a coaxial configuration the induced power includes mutual interference) for various lift-offset values at 80, 120, and 160 knots. For this calculation, the pilot collective (equal upper and lower rotor collective pitch) was progressively increased up to and through stall for a zero shaft angle. Operating condition is $6000 \mathrm{ft} / 95^{\circ} \mathrm{F}$ and rotor blade tip speed is $650 \mathrm{ft} / \mathrm{s}$. The trim solution solves for the rotor cyclic pitch to achieve the target hub roll moment (lift offset) and zero hub pitch moment for each rotor. Lift offset is the effective lateral displacement of the lift vector for each of the rotors from the hub center. The lift offset is defined as $\Delta M_{x} / L R$, where $\Delta M_{x}$ is the differential rotor roll moment, $L$ is the sum of the lift of both rotors. The net hub roll moment is zero. The lift-offset values are referenced to 1-g flight and hub moment values are maintained constant as collective increases.

The analysis shows a moderate increase in the rotor induced plus profile power without stall. As stall becomes important, the slope of the power curve quickly flattens. The maximum lift capability of the rotor significantly increases as the lift offset increases. At 80 knots (Fig. 3(a)), the maximum $C_{L} / \sigma$ is around 0.16 with zero lift offset and reaches the maximum value of 0.25 with the lift offset of 0.5 . Further increase of the lift offset to 0.6 decreases the maximum lift capability (Fig. 3(b)). At a higher speed of 120 knots, the maximum $C_{L} / \sigma$ keeps increasing up to the lift offset of 0.6 and reaches the maximum value of 0.27 (Fig. 3(c)). At 160 knots, the maximum $C_{L} / \sigma$ of 0.30 was obtained with the lift offset of 0.8 (Fig. 3(f)). The maximum lift capability of the lift-offset rotor extends well beyond the McHugh lift boundary (Refs. 16-18). Comparison between the present rotor results and McHugh's rotor test data will be made later in this section. The benefit of larger lift offset occurs at higher thrust and power. In practice, larger lift offset (larger hub roll moment) might be prohibited by structural weight (blades, hub, shaft, etc), drag, and the blade tip clearance between the two rotors.

Figure 4 shows the upper and lower rotor induced power plus profile power versus rotor lift at 120 knots. The analysis results are the same as those in Fig. 3(c), except that the individual rotor performance is plotted. In general, the two rotors exhibit very similar trends. Both upper and lower rotors show almost identical rotor performance without lift offset. However, the lower rotor shows slightly higher maximum lift capability than the upper rotor as the lift offset increases. Although not shown here, the same trend was observed for 80 and 160 knots. The higher maximum lift capability of the lower rotor is caused by the interference between the two rotors, as shown by detailed airloads results presented in the next section.

Figure 5 shows the upper and lower rotor lift and torque as a function of collective at 120 knots with various lift-offset values. As Fig. 5(a) shows, the two rotors generate almost identical lift for a given collective without lift offset. As lift offset increases, the lower rotor generates more lift than the upper rotor for the same collective, due to interference effect. Without interference, each rotor generates an identical amount of lift regardless of lift offset. Interference decreases lift produced by both rotors, but the reduction is slightly higher for the upper rotor. The maximum lift of the lower rotor is about $2.7 \%$ and $6.0 \%$ higher than that of the upper rotor for the liftoffset values of 0.2 and 0.4 , respectively. Figure 5(b) shows the upper and lower rotor torque with lift-offset values of 0.0 and 0.4. The results with the lift-offset value of 0.2 is not included for clarity. Although torque balance was not attempted in these calculations, the required torque between the upper and lower rotor is very close without lift offset. As lift offset increases, the lower rotor required slightly more torque (and thus power).

Figure 6 shows the rotor lift and induced power plus profile power as a function of collective at 120 knots. As lift offset increases, the rotor lift increases almost linearly for the same collective and the maximum lift generated by the rotor also increases. The trend for the rotor induced power plus profile power is different than that of the rotor lift. The power grows quadratically as a function of lift offset. For small lift-offset values (e.g. 0.1 and 0.2 ), there is a small increase in the rotor induced power plus profile power. Thus, the rotor generates significantly more lift for a small power penalty.

Figure 7 shows the effects of shaft angle on rotor performance for the lift-offset value of 0.2. The shaft angle was varied $\pm 4^{\circ}$. The shaft angle variation has a negligible influence at 80 knots, but its effect is larger at higher speeds. The forward shaft tilt $\left(\alpha_{s}=-4^{\circ}\right)$ reduces and aft shaft tilt $\left(\alpha_{s}=\right.$ $+4^{\circ}$ ) increases the maximum lift capability at 120 and 160 knots. The increase in rotor lift with a positive shaft angle is produced at the cost of an increase in drag, which must be overcome by the propeller in the current configuration.

The rotor performance for the coaxial rotor (lift offset $=$ 
0.0) is calculated and compared with that of the McHugh rotor, as shown in Fig. 8. The McHugh rotor test was performed with a 1/10-scale $\mathrm{CH}-47 \mathrm{~B} / \mathrm{C}$ type 4-bladed articulated rotor which has a V23010-1.58 airfoil section and a linear twist of -7 degrees (Refs. 16-18). The rotor was designed with sufficient structural strength that the true aerodynamic limits were obtained. A parametric study was conducted to examine and understand the differences between the present coaxial rotor and the McHugh rotor in terms of maximum lift capabilities. The parameters investigated in this study are airfoils, taper, and twist. The effects of those parameters on the prediction of rotor lift were examined by replacing the coaxial rotor quantities with the McHugh rotor quantities.

Figure 8(a) shows the parametric study results for airfoil, taper, and twist variations at 120 knots. First, the coaxial rotor was modified to a 8-bladed single main rotor with the same solidity. The lift generated by the single rotor is almost identical to that by the coaxial rotor. Next, the present state-of-the-art airfoils were replaced by the full-scale V23010 airfoil along the entire blade span for the single rotor. This airfoil change substantially reduced rotor lift, especially at high collective angles. This result shows that the present airfoils have superior stall characteristics than the V23010 airfoil. For comparison with model-scale test data, correcting the full-scale airfoil test data for Reynolds number effects is necessary. The Reynolds number correction, as Reynolds number is reduced for the model-scale rotor, increased drag at all angles of attack and Mach numbers. The Reynolds number correction for lift and moment was made by reducing static stall angles of attack. Detailed Reynolds number correction procedure and the lift, drag, and moment coefficients of the full-scale and modelscale V23010 airfoils are available in Reference 15 . The model-scale V23010 airfoil with Reynolds number correction further reduced rotor lift at all collective angles. Subsequently, taper was removed and nonlinear twist was changed to $-7 \mathrm{deg}$ linear twist to match with the McHugh rotor blade in addition to the model-scale V23010 airfoil. Again, these changes decreased rotor lift.

One last modification was to reduce the number of blades to four and the results were directly compared with the McHugh rotor data and analysis in Fig. 8(b). The change in number of blades slightly increased rotor lift compared to the 8-bladed rotor results shown in Fig. 8(a). The McHugh rotor test data shown in the figure were obtained at advance ratios of 0.2 and 0.3 . In the wind tunnel test, a sweep in rotor lift coefficient was made at a fixed rotor propulsive force coefficient $\left(X / q d^{2} \sigma=0.05\right)$ by increasing shaft angle and collective at the required advance ratio and tip Mach number $\left(V_{T I P}\right.$ $=620 \mathrm{ft} / \mathrm{s}$ ). The analysis was conducted with CAMRAD II and the results were compared with the test data. For the analytical calculations, full-scale $\mathrm{CH}-47 \mathrm{~B}$ blade properties were obtained from the Boeing Company and were scaled to the model configuration. The Reynolds number-corrected airfoil decks were used for the performance analysis. A free wake and unsteady aerodynamics were used, but a dynamic stall model was not incorporated. There is good agreement between the analysis and measurement, which shows the validity of the analysis tool used for the present study. The final modified case (4-bladed single rotor with model-scale V23010 airfoil, no taper, $-7 \mathrm{deg}$ linear twist) at $\mu=0.31$ shows similar results to the McHugh rotor test data and analysis. There are still remaining differences between the two cases as hub type (articulated vs. hingeless), blade dynamics (natural frequencies), tip speed $(620 \mathrm{ft} / \mathrm{s}$ vs. $650 \mathrm{ft} / \mathrm{s})$, propulsive trim $\left(X / q d^{2} \sigma=0.05\right.$ vs. $\left.X / q d^{2} \sigma=0.0\right)$, etc. However, the combined effects of these remaining differences appear to be small for the performance calculations.

Figure 9 shows the maximum lift capability of coaxial, liftoffset rotors as a function of advance ratio. The McHugh's lift boundary was also plotted to compare with the conventional rotor lift limit. The present coaxial rotor shows substantially higher maximum lift even without lift offset; about $16 \%$ and $38 \%$ higher at $\mu=0.21$ and 0.42 , respectively. The maximum lift capability of the coaxial rotor decreases with speed, but much less than that of the conventional rotor. As lift offset increases, the maximum lift capability of the rotor significantly increases. The maximum $C_{L} / \sigma$ is 0.25 at 80 knots $(\mu$ $=0.21), 0.27$ at 120 knots $(\mu=0.31)$, and 0.30 at 160 knots ( $\mu=0.41$, with the lift-offset values of $0.5,0.6$, and 0.8 , respectively. As mentioned in the introduction, the $\mathrm{ABC}$ rotor system in the XH-59A flight tests demonstrated up to $C_{T} / \sigma=$ 0.28 in maneuvering flight (Ref. 3). The lift-offset value used for that maneuvering flight is not documented. Although there are many differences between the present rotor and the $\mathrm{ABC}$ rotor such as number of blades, airfoils, planform, etc., the present analysis shows a similar high-lift capability of coaxial, lift-offset rotor.

\section{Rotor control}

Figure 10 shows the cyclic angles of the upper rotor as a function of collective for various lift-offset values at 120 knots. Although not shown here, the lower rotor operated almost at the same cyclic angles as the upper rotor. At forward flight, the relative wind speed encountered by the advancing blade is larger than the relative wind speed acting on the retreating blade. As a result of the relative wind speed, the advancing side produces more lift than the retreating side. In order to maintain roll moment trim, longitudinal cyclic is required to decrease pitch of the advancing blade and increase pitch of the retreating blade. As collective increases, this lift imbalance increases and thus more longitudinal cyclic (negative) is required. Figure 10(a) shows progressively higher longitudinal cyclic requirement for trim as collective increases for all the lift-offset values used. By operating a rotor with lift offset, the advancing side can carry more lift than the retreating side of the rotor disk. And the required longitudinal cyclic angle for trim decreases (less negative) as the lift-offset value increases. Figure 10(b) shows that the lateral cyclic control required to trim hub pitching moment to zero increases with collective, as expected. The lift offset also increases the required lateral cyclic angle. Figure 10(c) shows the swashplate tilt angle, which is a combination of the longitudinal and lateral cyclic angles. The swashplate tilt angle increases almost 
linearly for zero lift offset as collective increases. As lift offset increases, the swashplate tilt angle varies quadratically. At low collective angles, the swashplate tilt angle decreases initially and then increases as lift offset increases due to the sign change of longitudinal cyclic. At high collective angles, the swashplate tilt angle decreases as lift offset increases due to the reduced longitudinal cyclic angle requirement to trim.

\section{Blade airloads and structural loads}

Lift offset has a significant influence on rotor blade loads as well as performance. This section examines the effects of lift offset on rotor blade airloads and structural loads for mostly high-collective (thus high lift) cases.

Figures 11, 12, and 13 show nondimensional blade section lift at 90-deg and 270-deg azimuth for both upper and lower rotors for 15-deg collective at 80, 120, and 160 knots, respectively. Airloads results with three different lift-offset values $(0.0,0.2$, and 0.4$)$ are plotted with and without interference effects between the two rotors. CAMRAD II has an option to suppress the mutual interference effects between the wakes generated by the rotors.

Figure 11 shows blade section lift at 80 knots. The retreating side of each rotor does not generate any lift inboard due to reverse flow, but generates positive lift from the mid to tip of each blade. The section lift on the retreating side is insensitive to the lift offset. On the advancing side, both upper and lower rotor blades generate positive lift. With lift offset (carrying more roll moment), the advancing side of each rotor generates significantly more lift, exploring the lift potential of the advancing blades. The interference between the two rotors has a small influence on the retreating side regardless of lift-offset value. Without interference, both upper and lower rotors exhibit similar lift distributions as both rotors need to achieve the same trim targets for the same collective. Inclusion of the interference effects decreases section lift of the upper rotor blade on the advancing side for an entire blade span, which is counter intuitive to the hover case. The interference has more influence on the lift distribution of the lower rotor, especially on the advancing side. The interference reduces section lift on the mid span, but increases section lift near the blade tip of the lower rotor.

At higher speeds, as shown in Figs. 12 and 13, zero section lift on the retreating side of each rotor blade expands to mid span as the reverse flow region increases. On the advancing side, the section lift is positive inboard and negative outboard for the zero lift-offset case in order to maintain roll moment trim, which is a typical aerodynamic behavior at high speed flight. Again, with lift offset, the advancing side of each rotor generates significantly more lift, and the lift potential of the advancing blades is utilized. Although not shown here, a lift offset of 0.6 further increased section lift on the advancing side at these speeds and collective. Inclusion of the interference effects decreases section lift of the upper rotor on the advancing side, and increases section lift of the lower rotor on the retreating side.
Figures 14, 15, and 16 show the half peak-to-peak blade structural loads at $10 \%$ radius for the upper rotor as a function of collective at 80,120, and 160 knots, respectively. For the hingeless rotor blade, maximum blade loading occurs near the root and decreases along the blade toward the tip. The blade structural loads are shown in the nondimensional form and nondimensional flap bending moment is defined as:

$$
C_{F B M} / \sigma=\frac{M_{F}}{\rho N_{b} c \Omega^{2} R^{4}}
$$

where $M_{F}$ is the flap bending moment, $N_{b}$ is the number of blades, and $c$ is the nominal blade chord, $\Omega$ is the rotor speed, and $R$ is the radius. As mentioned before, the blade inertial and structural properties were scaled from the compound helicopter blade design of Ref. 10, not designed for the current configuration. Thus, absolute loads values are not meaningful but behavior can be examined. Without lift offset, all the blade structural loads components increase with collective, as expected. With lift offset, flap bending moment increases substantially to carry the hub roll moment even at low collective values. The magnitude of flap bending moment is dictated by lift-offset value (hub roll moment) but, is less sensitive to collective. Thus, the magnitude of flap bending moment with 0.2 lift offset is lower than that with zero lift offset at high collective values. The magnitude of flap bending moment is not much affected by speed. Chord bending moment increases with collective regardless of lift-offset, but is not affected much by speed. However, their magnitudes decrease with lift offset, especially at high collectives. The reason for the reduction appears to be reduced stall as lift offset increases. Torsion moments also increase with collective regardless of lift offset and increase with speed as well. However, their magnitudes slightly decrease with lift offset.

Figures 17(a) and 17(b) show the flap bending moment at $10 \%$ radius for the upper rotor blade at 120 knots for collective of $8 \mathrm{deg}$ and $15 \mathrm{deg}$, respectively. The vertical axes have the same range in order to compare the magnitudes between the two cases. There is a substantial increase in magnitude as collective increases for the zero lift-offset case. As lift offset increases, there is a strong amplitude change on both advancing and retreating sides in order to generate the hub roll moment. The peak-to-peak magnitude does not change with collective for non-zero lift-offset cases.

\section{CONCLUSIONS}

Maximum blade loading capability of coaxial, lift-offset rotors was investigated using a rotorcraft configuration designed in the context of short-haul, medium-size civil and military missions. The pilot collective (equal upper and lower rotor collective pitch) was progressively increased up to and through stall with the shaft angle set to zero. The trim solution solves for the rotor cyclic pitch to achieve the target hub roll moment (lift offset) and zero hub pitch moment for each rotor. The effects of lift offset on rotor lift and power, controls, and blade airloads and structural loads are examined. From this study the following conclusions are obtained: 
1) The maximum lift capability of the coaxial rotor increases as lift offset increases and extends well beyond the McHugh lift boundary as the lift potential of the advancing blades is fully realized. The maximum $C_{L} / \sigma$ achieved by the coaxial, lift-offset rotor is 0.25 at 80 knots $(\mu=0.21), 0.27$ at 120 knots $(\mu=0.31)$, and 0.30 at 160 knots $(\mu=0.41)$, with the lift-offset values of $0.5,0.6$, and 0.8 , respectively.

2) There is good agreement between the analysis and McHugh's rotor test data, which shows the validity of the analysis tool and approach used for the present study.

3) Parametric study shows that airfoils, taper, and twist play important roles in defining the maximum lift capability of the lift-offset rotors.

4) The advancing side of each rotor generates significantly more lift with lift offset. However, the section lift on the retreating side is insensitive to lift offset.

5) Without lift offset, all the blade structural moments increase with collective, as expected. With lift offset, flap bending moment increases substantially to carry a hub roll moment even at low collective values. The magnitude of flap bending moment is dictated by lift-offset value (hub roll moment) but, is less sensitive to collective and speed.

\section{REFERENCES}

${ }^{1}$ Ruddell, A. J., and Macrino, J. A., "Advancing Blade Concept (ABC) High Speed Development," American Helicopter Society 36th Annual Forum, Washington, D.C., May 13-14, 1980.

${ }^{2}$ Linden, A. W., and Ruddell, A. J., "An ABC Status Report," American Helicopter Society 37th Annual Forum, Washington, D.C., May 17-20, 1981.

${ }^{3}$ Ruddell, A. J., "XH-59A ABC Technology Demonstrator Altitude Expansion and Operational Tests," USAAVRADCOM TR 81-D-35, December 1981.

${ }^{4}$ Bagai, A., "Aerodynamic Design of the X2 Technology Demonstrator TM," American Helicopter Society 64th Annual Forum, Montreal, Canada, April 29-May 1, 2008.

${ }^{5}$ Blackwell, R., and Millott, T. A., "Dynamics Design Characteristics of the Sikorsky X2 Technology TM Demonstrator Aircraft," American Helicopter Society 64th Annual Forum, Montreal, Canada, April 29-May 1, 2008.

${ }^{6}$ Walsh, D., Weiner, S., Arifian, K., Bagai, A., Lawrence, T., and Blackwell, R., "Development Testing of the Sikorsky X2 Technology TM Demonstrator," American Helicopter Society 65th Annual Forum, Grapevine, TX, May 27-29, 2009.

${ }^{7}$ Walsh, D., Weiner, S., Arifian, K., Lawrence, T., Wilson, M., Millott, T., and Blackwell, R., "High Airspeed Testing of the Sikorsky X2 Technology TM Demonstrator," American Helicopter Society 67th Annual Forum, Virginia Beach, VA, May 3-5, 2011.
${ }^{8}$ Johnson, W., "Influence of Lift Offset on Rotorcraft Performance,” NASA TP 2009-215404, November 2009.

${ }^{9}$ Johnson, W., Moodie, A. M., and Yeo, H., "Design and Performance of Lift-Offset Rotorcraft for Short-Haul Missions," American Helicopter Society Future Vertical Lift Aircraft Design Conference, San Francisco, CA, January 18-20, 2012.

${ }^{10}$ Yeo, H., and Johnson, W., "Aeromechanics Analysis of a Heavy Lift Slowed-Rotor Compound Helicopter," Journal of Aircraft, Vol. 44, (2), March-April 2007.

${ }^{11}$ Johnson, W., "Technology Drivers in the Development of CAMRAD II," American Helicopter Society Aeromechanics Specialists Conference, San Francisco, CA, January 19-21, 1994.

${ }^{12}$ Yeo, H., Bousman, W. G., and Johnson, W., "Performance Analysis of a Utility Helicopter with Standard and Advanced Rotor," Journal of the American Helicopter Society, Vol. 49, (3), July 2004, pp. 250-270.

${ }^{13}$ Yeo, H., and Johnson, W., "Assessment of Comprehensive Analysis Calculation of Airloads on Helicopter Rotors," Journal of Aircraft, Vol. 42, (5), September-October 2005, pp. 1218-1228.

${ }^{14}$ Yeo, H., and Johnson, W., "Prediction of Rotor Structural Loads with Comprehensive Analysis," Journal of the American Helicopter Society, Vol. 53, (2), April 2008, pp. 193-209.

${ }^{15}$ Yeo, H., "Calculation of Rotor Performance and Loads Under Stalled Conditions," American Helicopter Society 59th Annual Forum, Phoenix, AZ, May 6-8, 2003.

${ }^{16} \mathrm{McHugh}, \mathrm{F}, \mathrm{J} .$, "What Are The Lift and Propulsive Force Limits At High Speed For The Conventional Rotor?," American Helicopter Society 34th Annual Forum, Washington, D.C., May 15-17, 1978.

${ }^{17}$ McHugh, F, J., Clark, R, and Solomon, M., "Wind Tunnel Investigation of Rotor Lift and Propulsive Force at High Speed - Data Analysis," NASA CR 145217-1, October 1977.

${ }^{18}$ McHugh, F, J., Clark, R, and Solomon, M., "Wind Tunnel Investigation of Rotor Lift and Propulsive Force at High Speed - Test Data Appendix," NASA CR 145217-2, October 1977. 
Table 1. Characteristics of the coaxial, lift-offset aircraft

\begin{tabular}{ll}
\hline \hline design gross weight, $\mathrm{lb}$ & 42491 \\
weight empty, $\mathrm{lb}$ & 29343 \\
design disk loading W/A, $\mathrm{lb} / \mathrm{ft}^{2}$ & 16 \\
design $C_{W} / \sigma$ & 0.08 \\
& \\
& 29.07 \\
rotor radius, $\mathrm{ft}$ & $2 \times 4$ \\
number blades & $2 \times 0.0991$ \\
solidity $\sigma$ (thrust-weighted) & 2.26 \\
chord (thrust-weighted), ft & upper rotor CCW, lower rotor CW \\
rotational direction & 725 (hover)/650(cruise) \\
tip speed, ft/s & \\
& 6.95 \\
propeller radius, ft & 0.1736 \\
propeller solidity $\sigma$ & 900 \\
tip speed, $\mathrm{ft} / \mathrm{s}$ & \\
\hline \hline
\end{tabular}



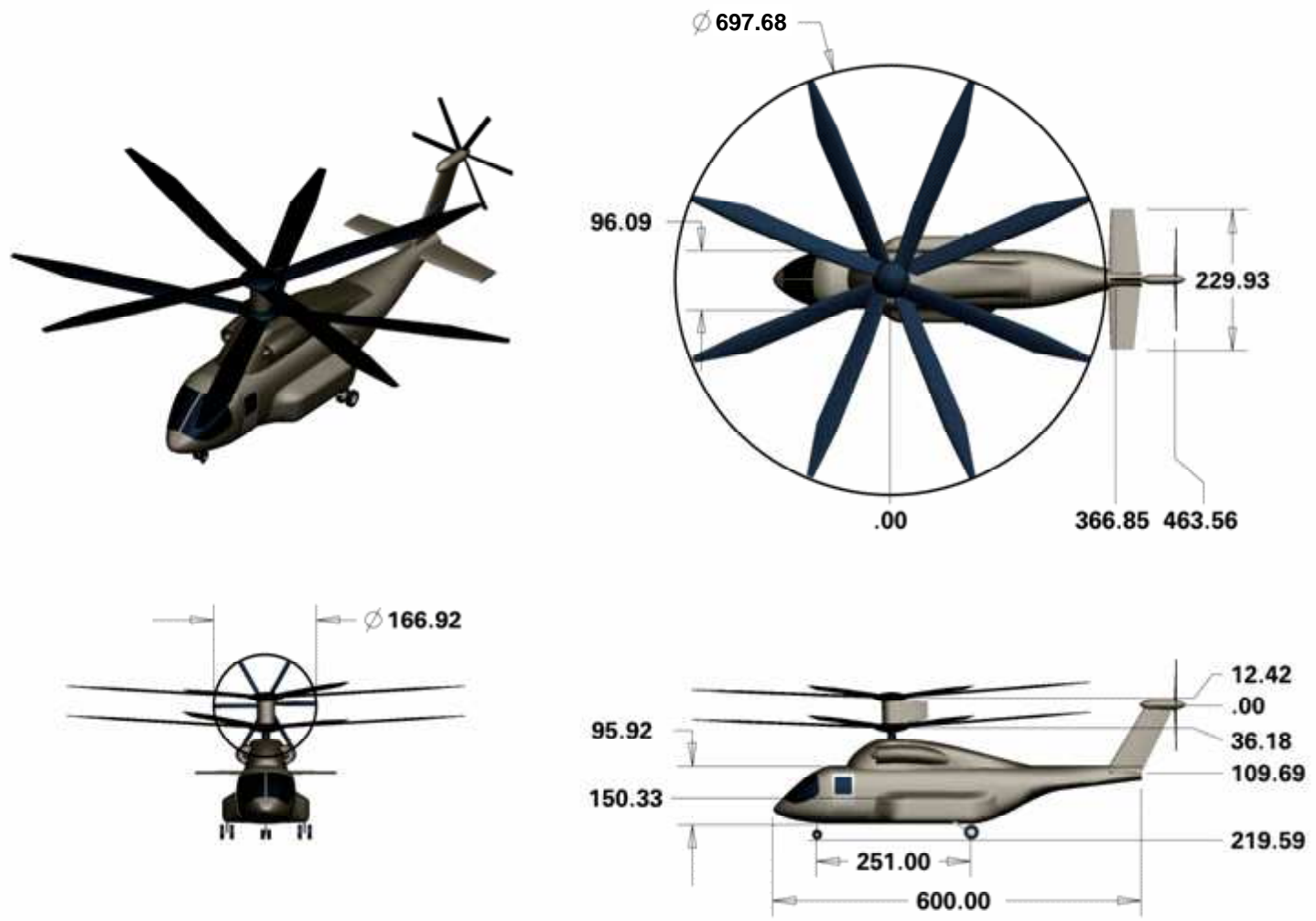

Fig. 1. Three-view drawing of the aircraft with coaxial, lift-offset rotors.
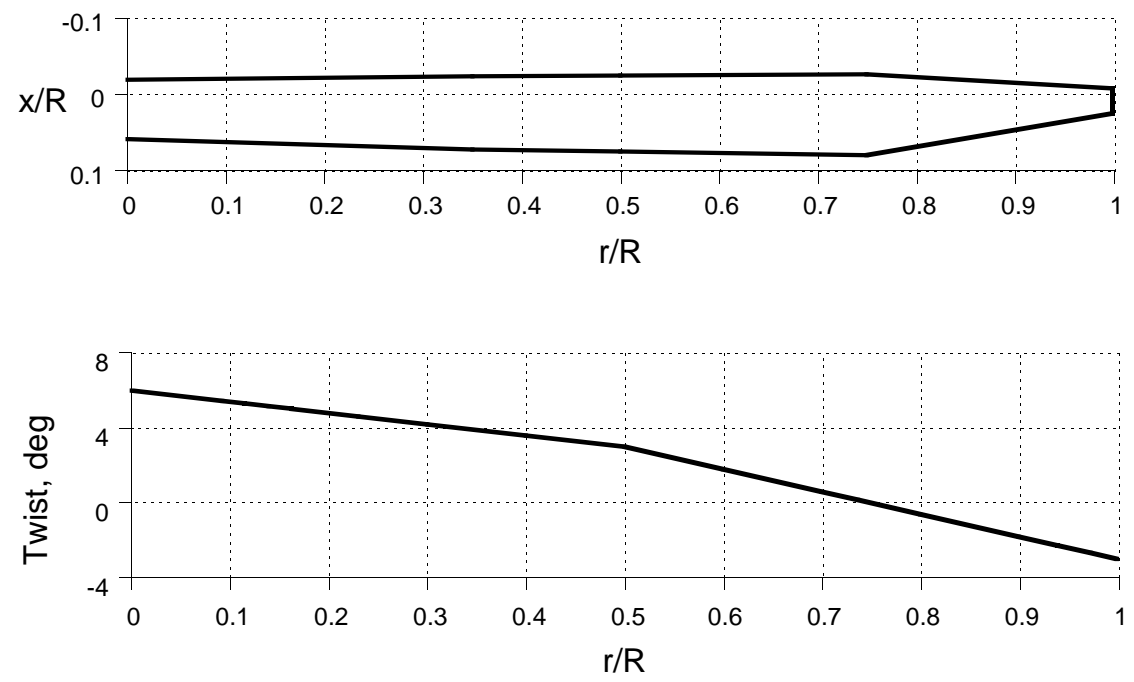

Fig. 2. Blade planform and twist 


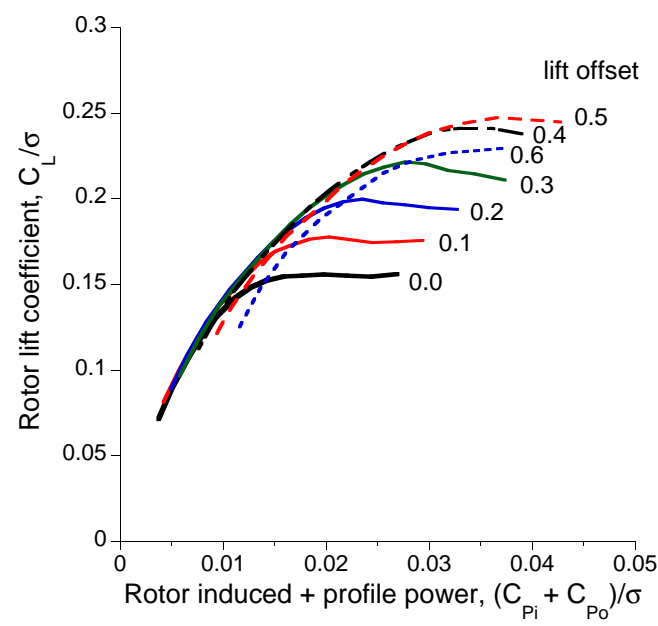

(a) 80 knots $(\mu=0.21)$

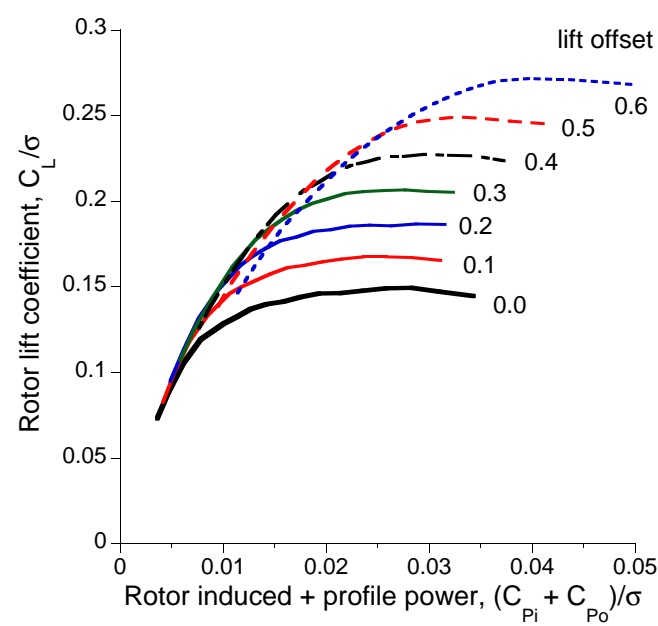

(c) 120 knots $(\mu=0.31)$

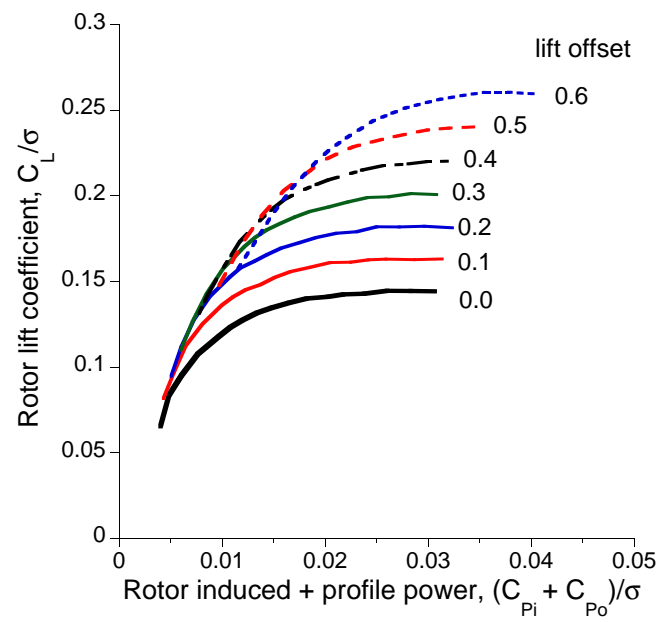

(e) 160 knots $(\mu=0.42)$

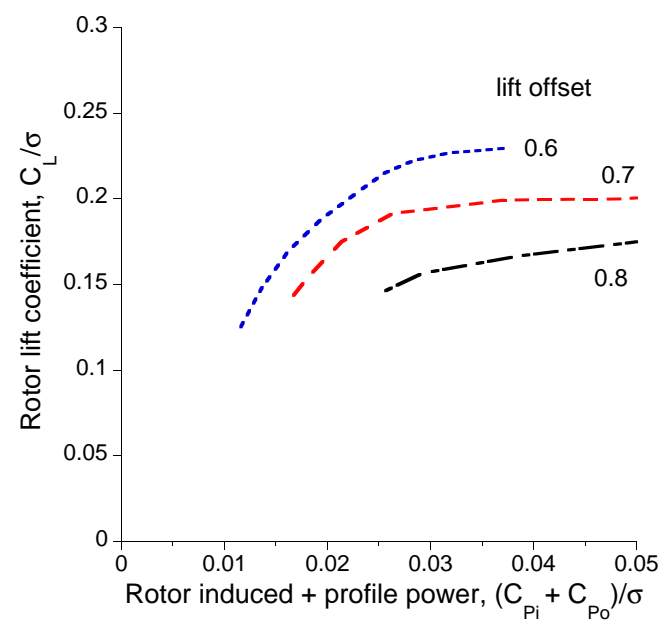

(b) 80 knots $(\mu=0.21)$

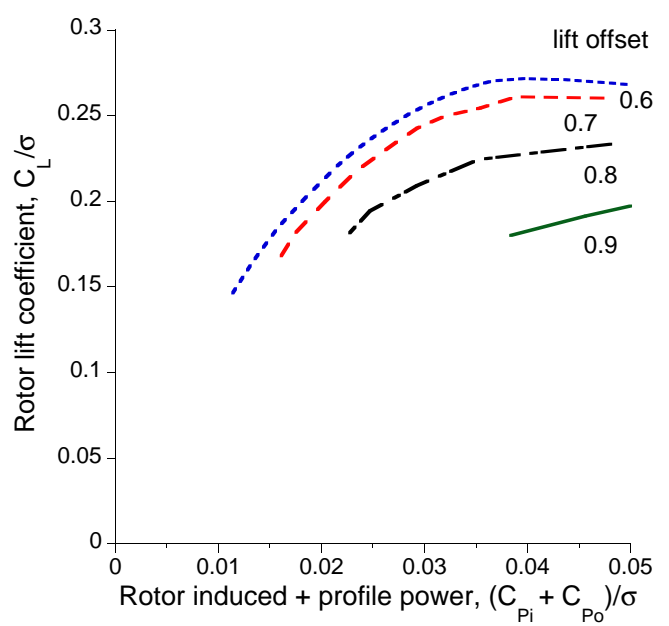

(d) 120 knots $(\mu=0.31)$

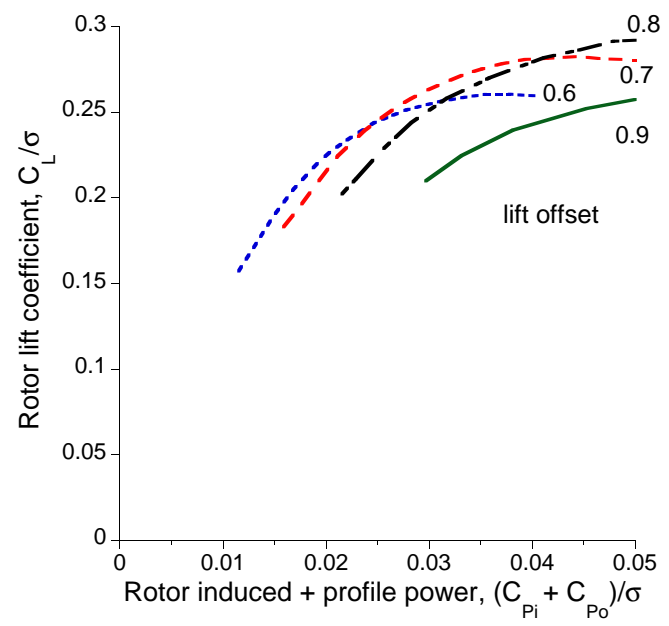

(f) 160 knots $(\mu=0.42)$

Fig. 3. Effect of lift offset on coaxial rotor performance, $\alpha_{s}=0^{\circ}$. 


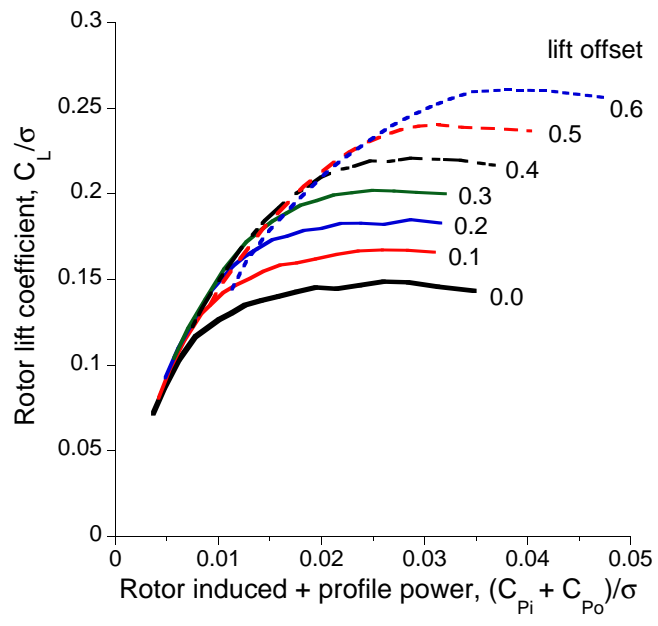

(a) upper rotor

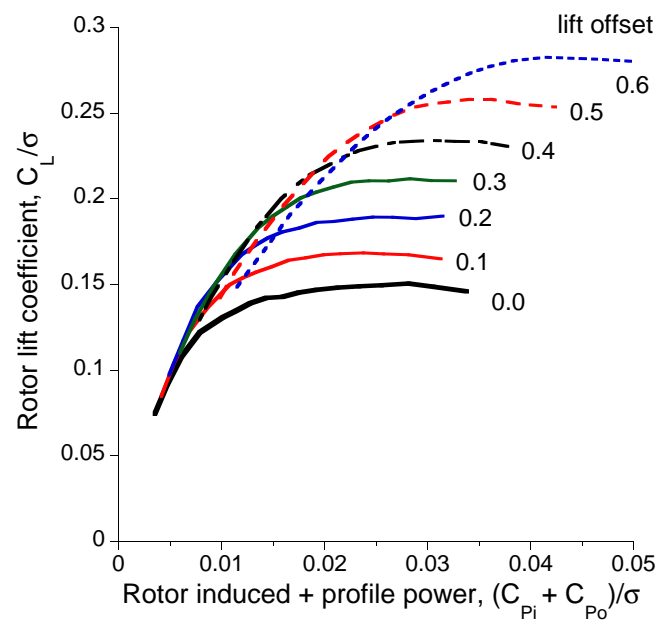

(b) lower rotor

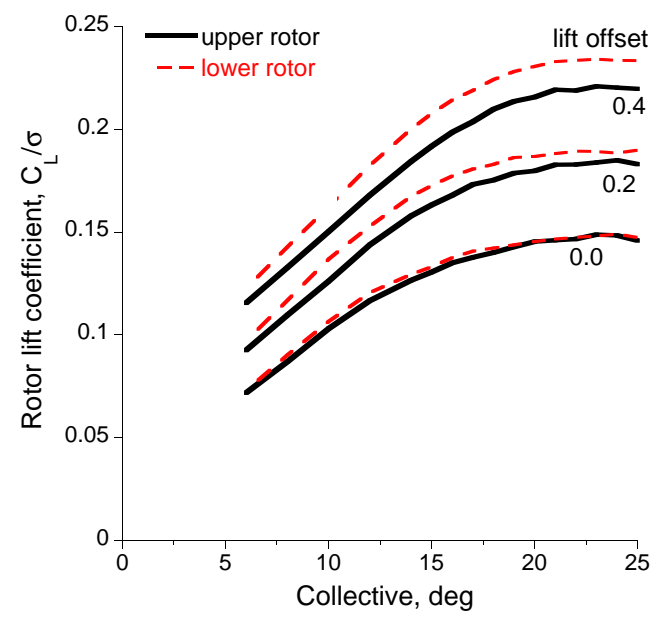

(a) lift

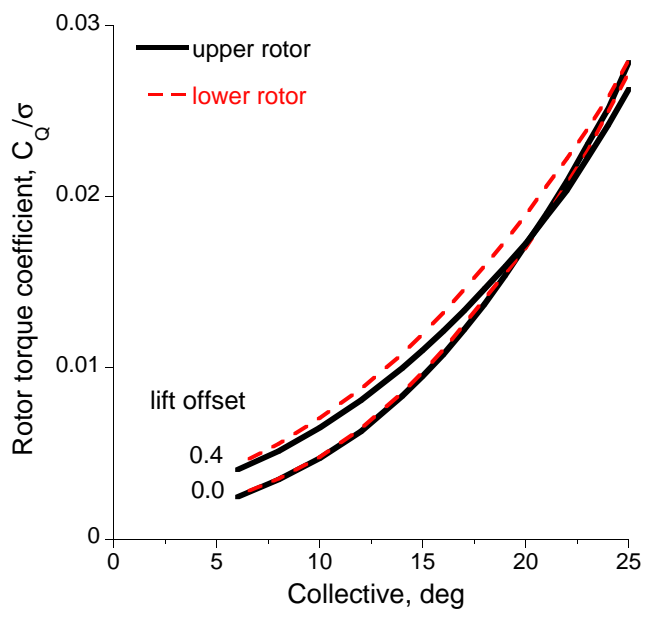

(b) torque
Fig. 4. Effect of lift offset on coaxial rotor performance, 120 knots $(\mu=0.31), \alpha_{s}=0^{\circ}$.
Fig. 5. Upper and lower rotor lift and torque of the coaxial rotor, 120 knots $(\mu=0.31), \alpha_{s}=0^{\circ}$. 


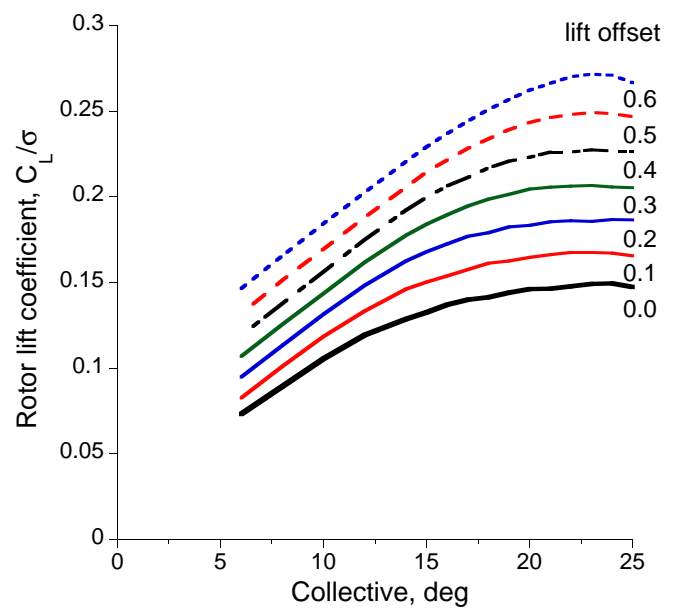

(a) rotor lift

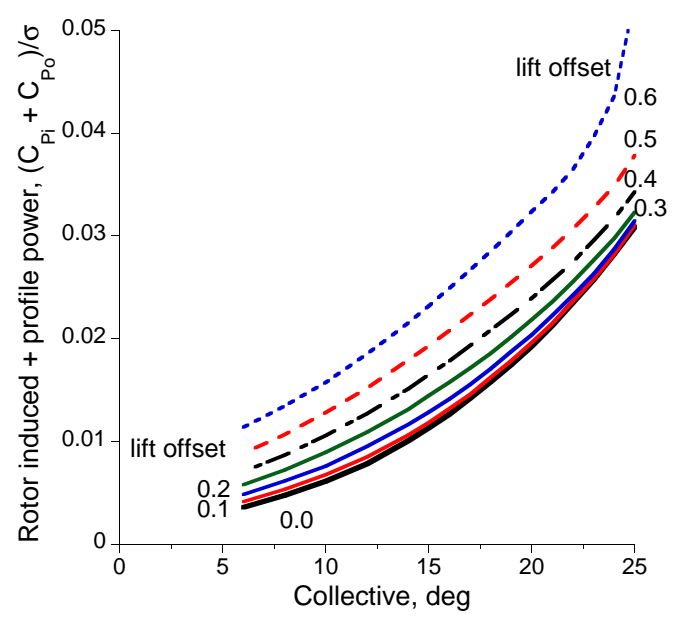

(b) rotor induced plus profile power

Fig. 6. Effect of lift offset on lift and power of the coaxial rotor, 120 knots $(\mu=0.31), \alpha_{s}=0^{\circ}$.

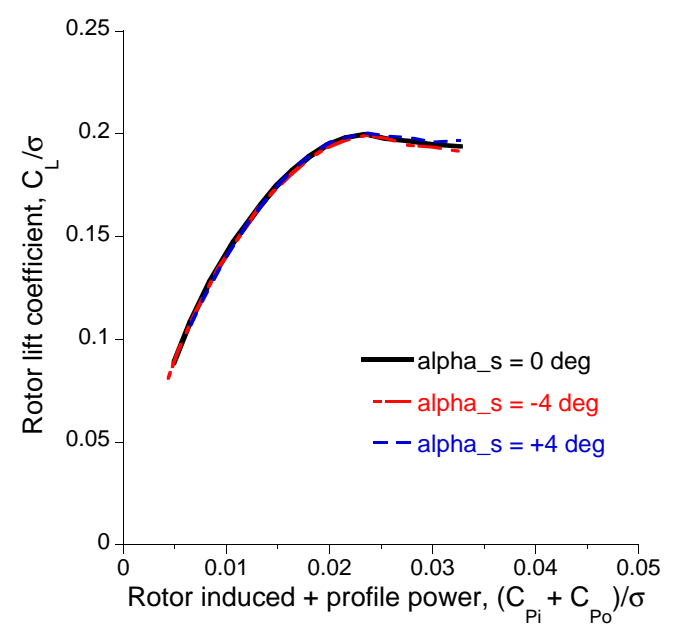

(a) 80 knots $(\mu=0.21)$

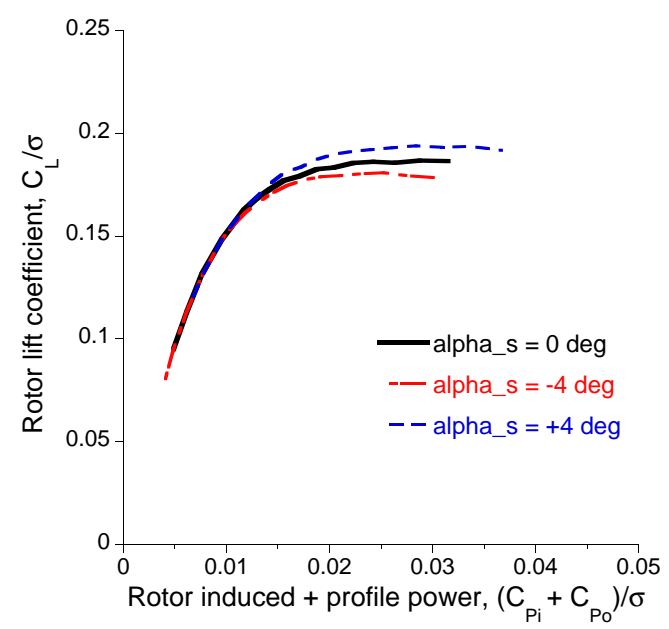

(b) 120 knots $(\mu=0.31)$

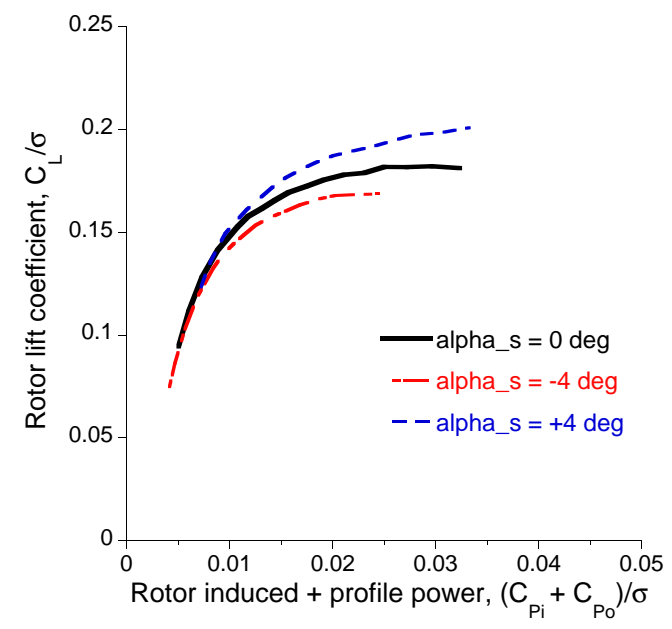

(c) 160 knots $(\mu=0.42)$

Fig. 7. Effect of shaft angle on coaxial rotor performance, lift offset $=0.2$. 


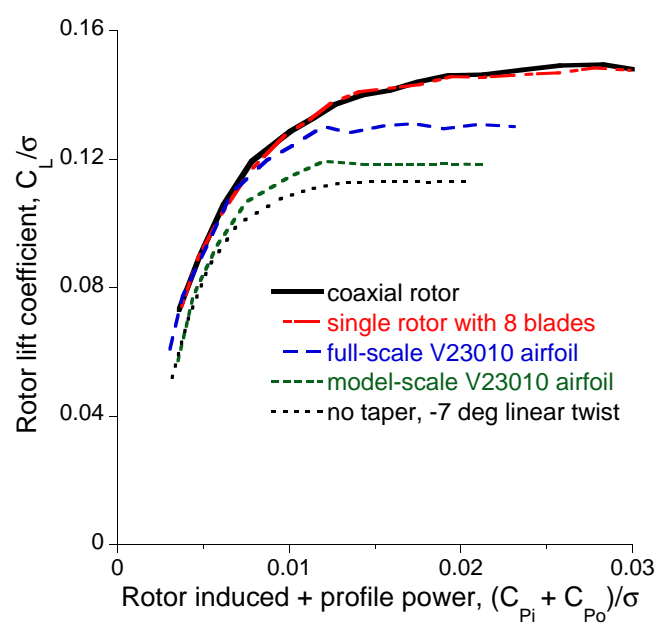

(a) 120 knots $(\mu=0.31), \alpha_{s}=0^{\circ}$

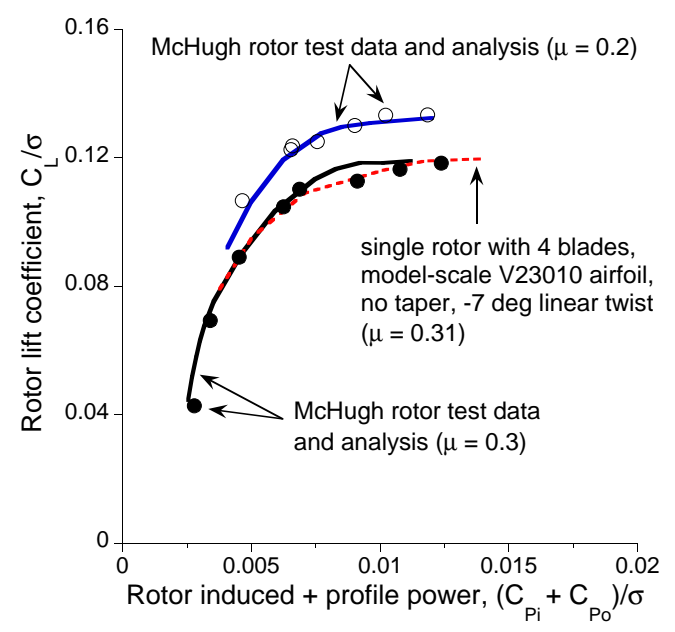

(b) McHugh rotor performance

Fig. 8. Comparison of rotor performance.

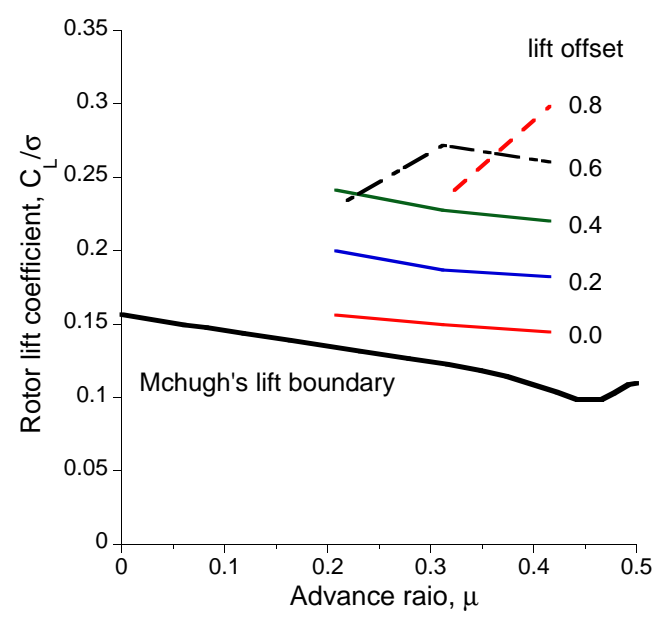

Fig. 9. Maximum lift capability of the coaxial rotor, $\alpha_{s}=$ $0^{\circ}$.

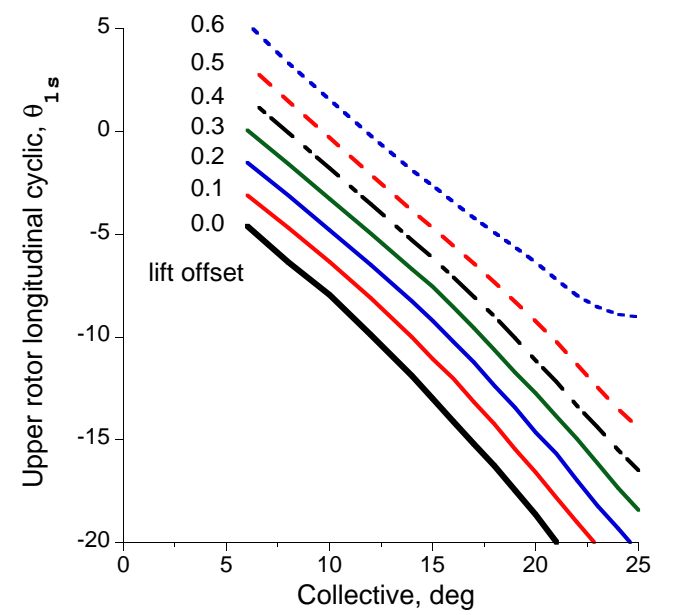

(a) longitudinal cyclic

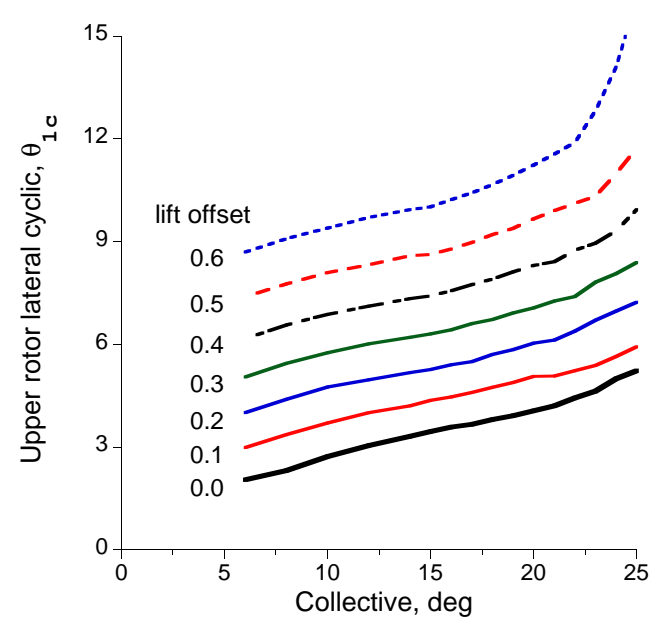

(b) lateral cyclic

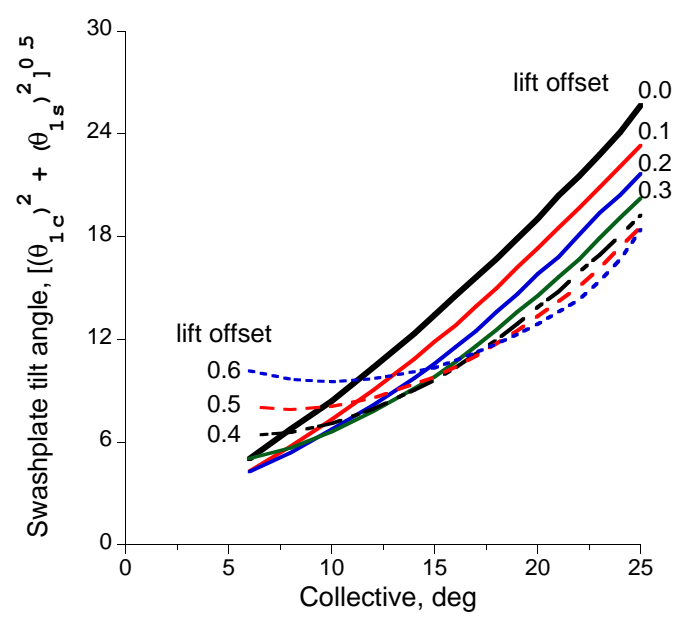

(c) Swashplate tilt angle

Fig. 10. Upper rotor cyclic control angles of the coaxial rotor, 120 knots $(\mu=0.31), \alpha_{S}=0^{\circ}$. 


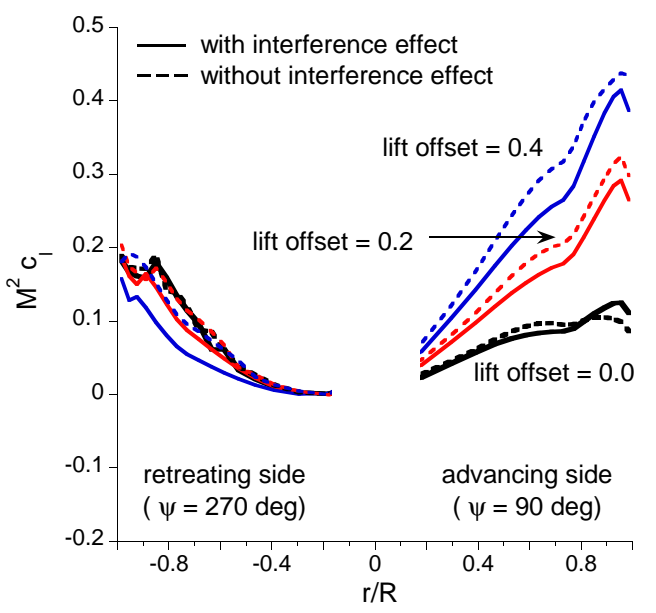

(a) upper rotor

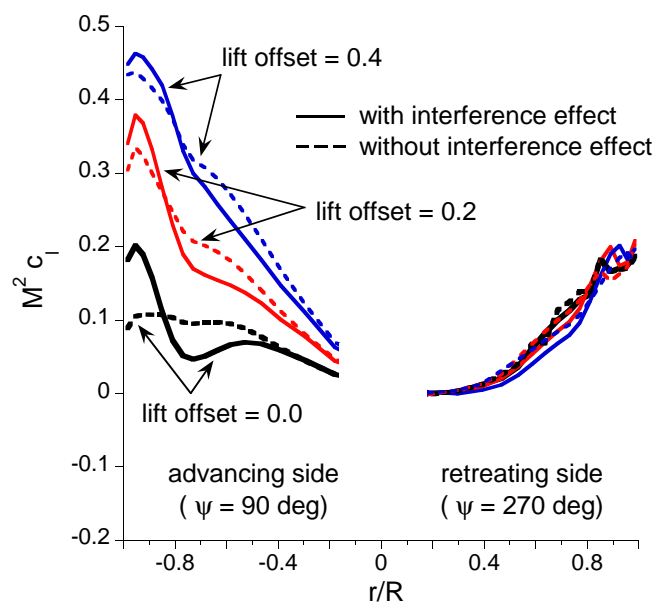

(b) lower rotor

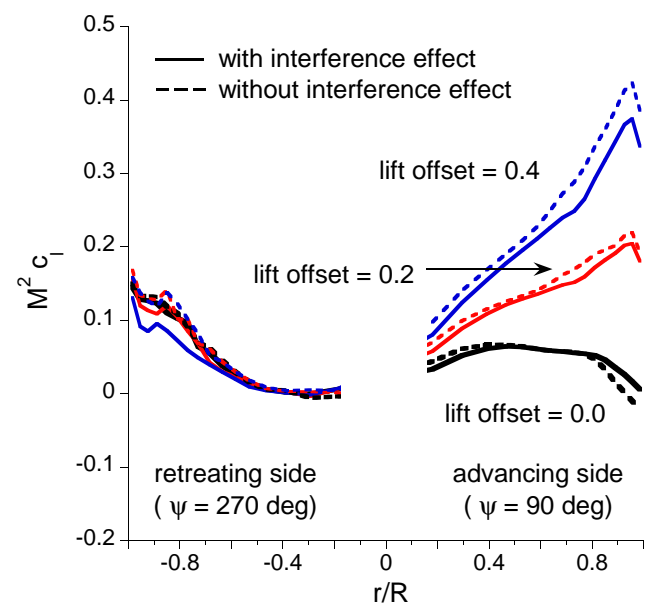

(a) upper rotor

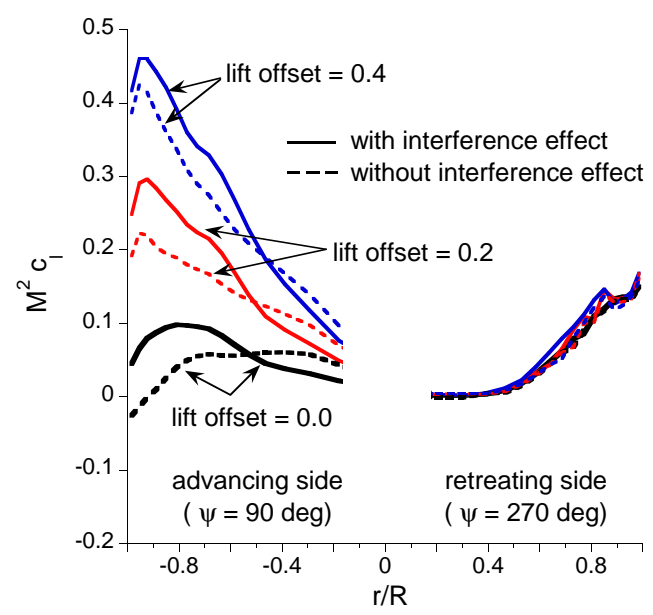

(b) lower rotor
Fig. 11. Blade section lift of the coaxial rotor, 80 knots $(\mu=$ $0.14), \alpha_{s}=\mathbf{0}^{\circ}, \mathbf{1 5}^{\circ}$ collective.
Fig. 12. Blade section lift of the coaxial rotor, 120 knots $(\mu=0.31), \alpha_{s}=\mathbf{0}^{\circ}, \mathbf{1 5}^{\circ}$ collective. 


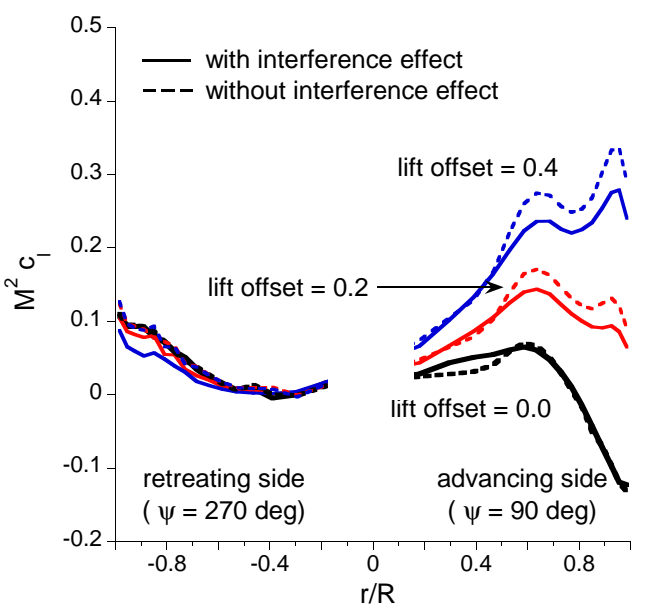

(a) upper rotor

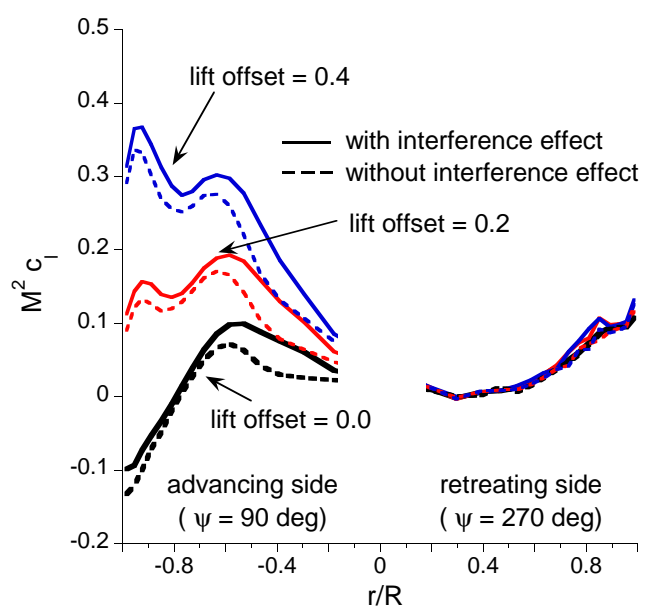

(b) lower rotor

Fig. 13. Blade section lift of the coaxial rotor, 160 knots $(\mu=0.42), \alpha_{s}=\mathbf{0}^{\circ}, \mathbf{1 5}^{\circ}$ collective.

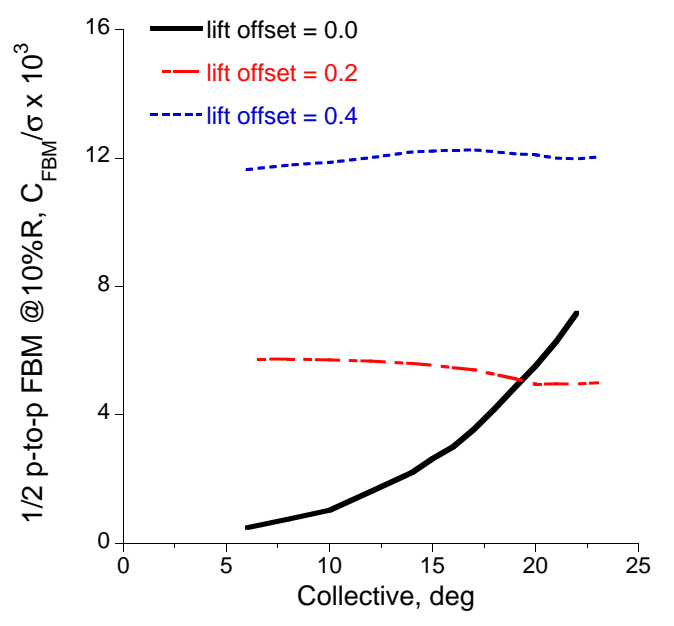

(a) Flap bending moment

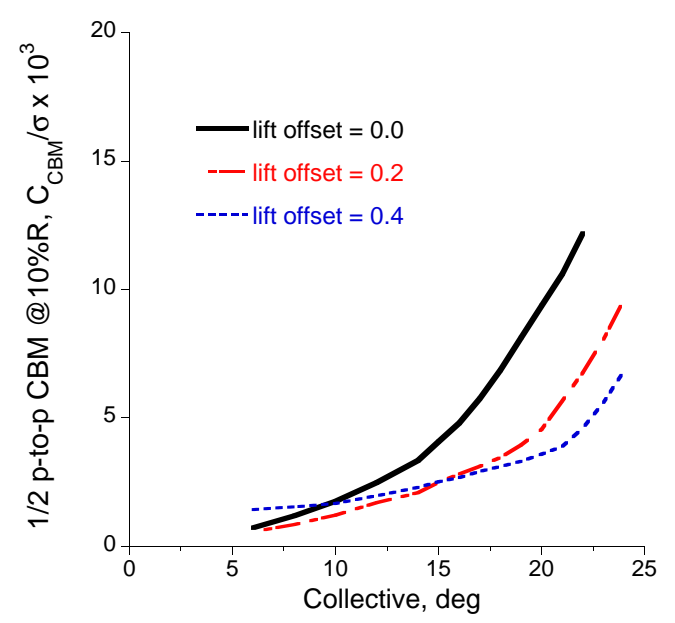

(b) Chord bending moment

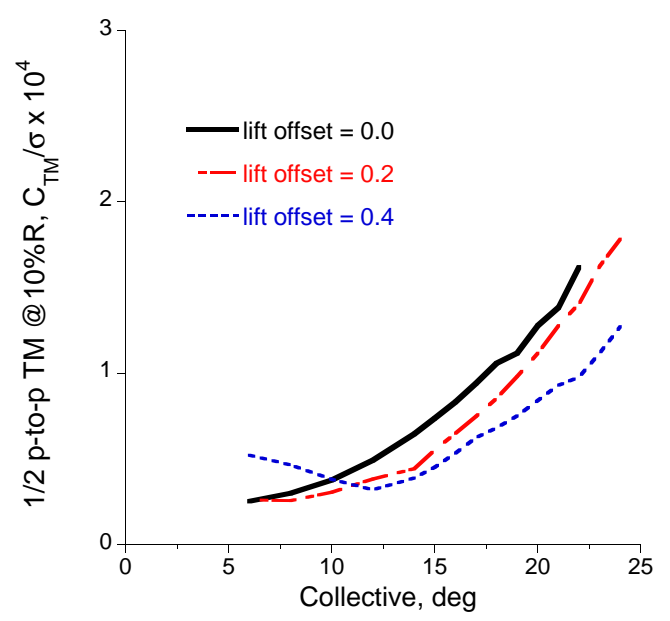

(c) Torsion moment

Fig. 14. Half peak-to-peak structural loads at $0.10 \mathrm{R}$ of the coaxial rotor, 80 knots $(\mu=0.14), \alpha_{s}=0^{\circ}$. 


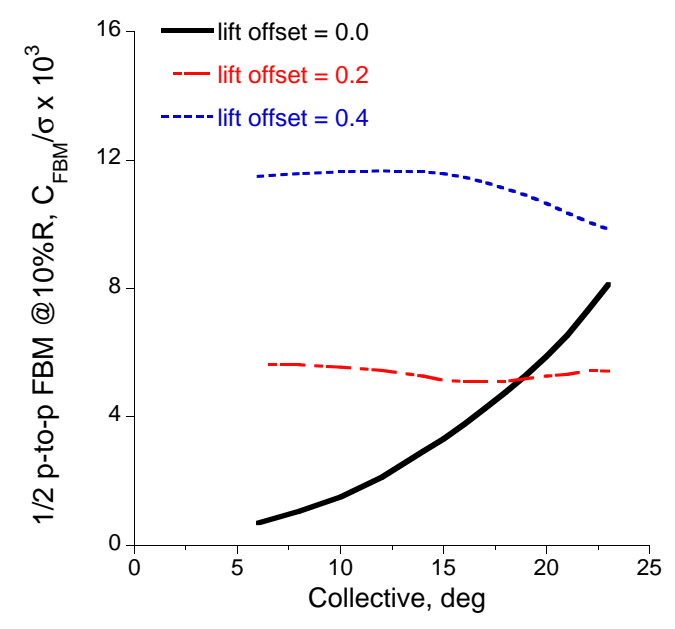

(a) Flap bending moment

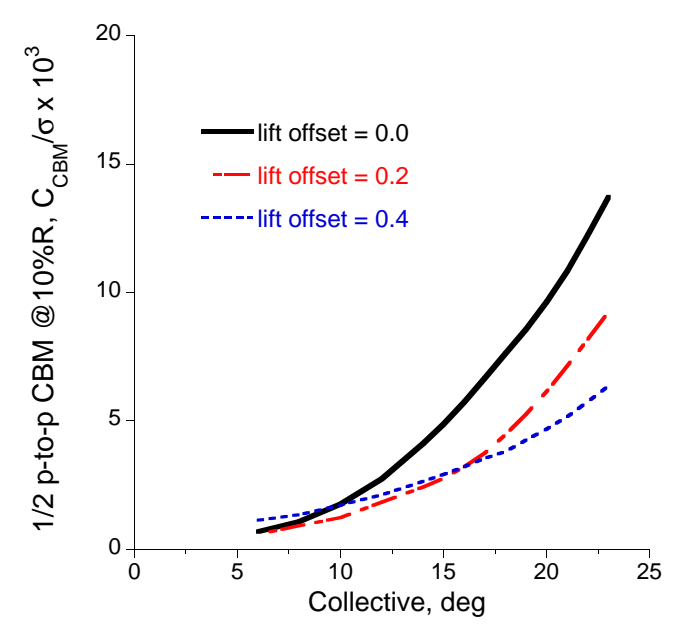

(b) Chord bending moment

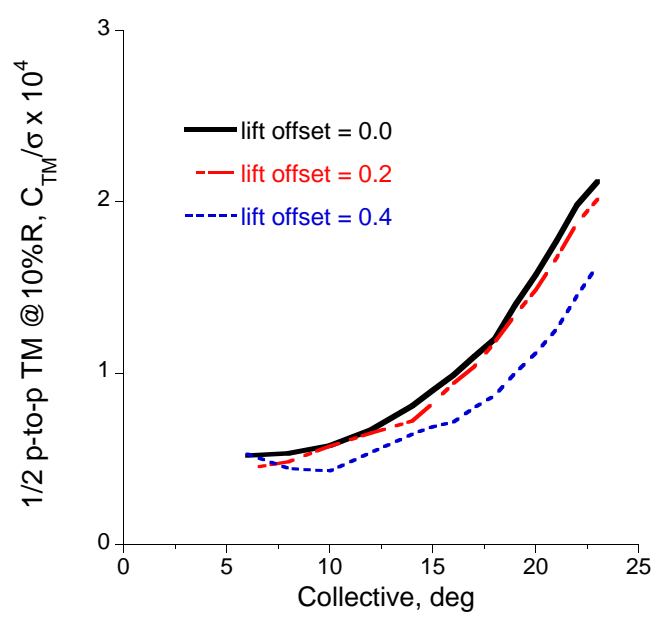

(c) Torsion moment

Fig. 15. Half peak-to-peak structural loads at $0.10 \mathrm{R}$ of the coaxial rotor, 120 knots $(\mu=0.31), \alpha_{s}=0^{\circ}$.

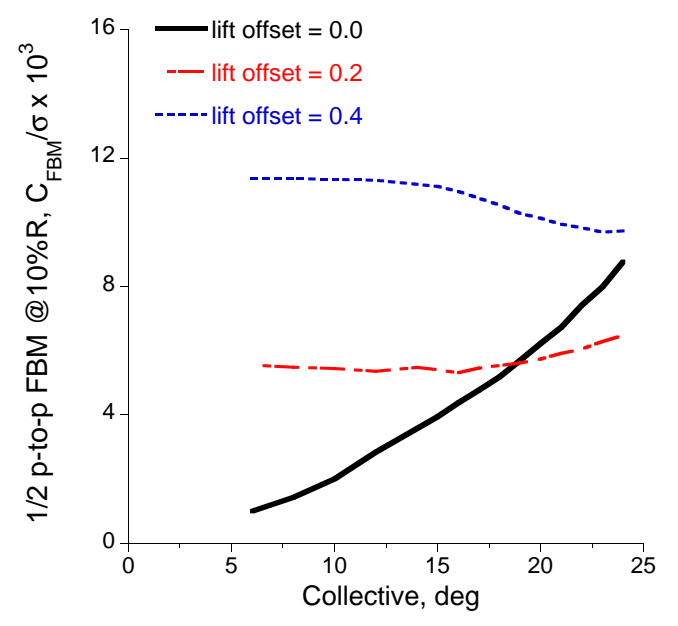

(a) Flap bending moment

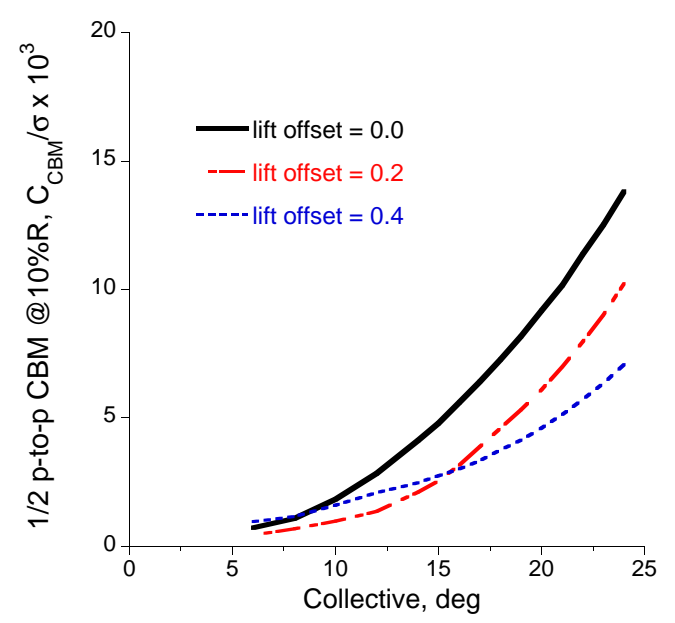

(b) Chord bending moment

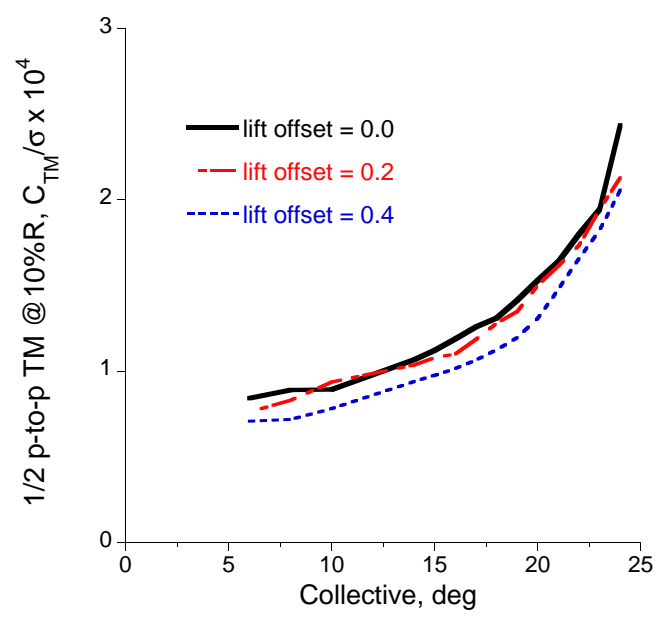

(c) Torsion moment
Fig. 16. Half peak-to-peak structural loads at $0.10 \mathrm{R}$ of the coaxial rotor, 160 knots $(\mu=0.42), \alpha_{s}=0^{\circ}$. 


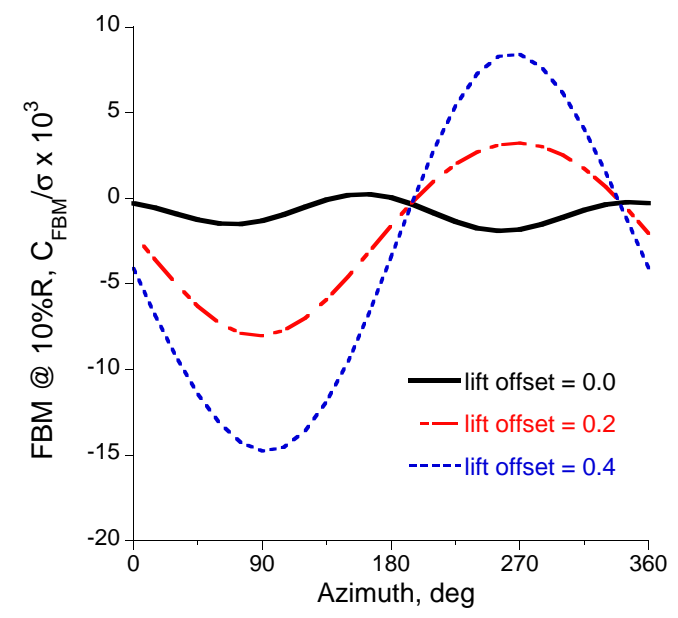

(a) collective $=8^{\circ}$

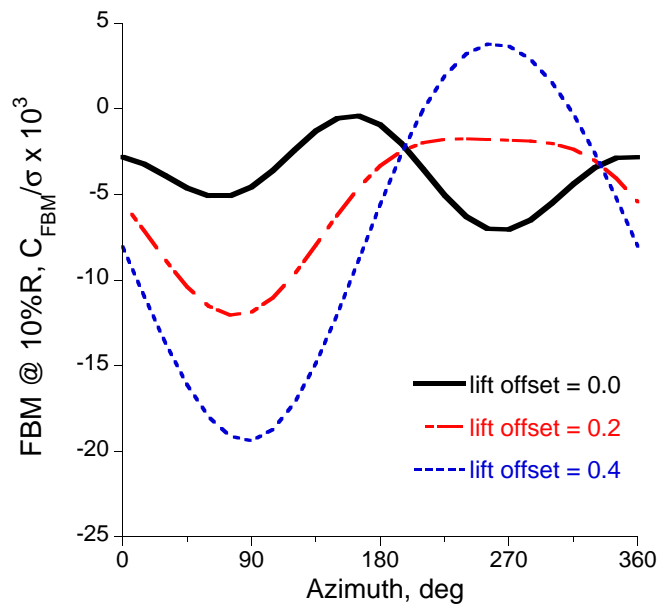

(b) collective $=15^{\circ}$

Fig. 17. Flap bending moment at $0.10 \mathrm{R}$ of the coaxial rotor, 120 knots $(\mu=0.31), \alpha_{s}=0^{\circ}$. 